\title{
Jointly Modeling Content, Social Network and Ratings for Explainable and Cold-start Recommendation
}

\author{
Ke $\mathrm{Ji}^{\mathrm{a}}$, Hong Shen ${ }^{\mathrm{a}, \mathrm{b}, \mathrm{c}}$ \\ ${ }^{a}$ School of Computer and Information Technology, Beijing Jiaotong University, Beijing, China \\ ${ }^{b}$ School of Information Science and Technology, Sun Yat-sen University, Guangzhou, China \\ ${ }^{c}$ School of Computer Science, University of Adelaide, Australia
}

\begin{abstract}
Model-based approach to collaborative filtering (CF), such as latent factor models, has improved both accuracy and efficiency of predictions on large and sparse dataset. However, most existing methods still face two major problems: (1) The recommendation results derived from user and item vectors of a set of unobserved factors are lack of explanation; (2) Cold start users and items out of user-item rating matrix cannot be handled accurately. In this paper, we propose a hybrid method for addressing the problems by incorporating content-based information (i.e, users' tags and items' keywords) and social information. The main idea behind our method is to build content association based on three factors-user interest in selected tags, tag-keyword relation and item correlation with extracted keywords, and then recommend the items with high similarity in content to users. Two novel methodsneighbor based approach and 3-factor matrix factorization are proposed for building tag-keyword relation matrix and learning user interest vector for selected tags and item correlation vector for extracted keywords. Besides, we introduce a social regularization term to help shape user interest vector. Analysis shows our method can generate explainable recommendation results with simple descriptions, and experiments on real dataset demonstrate our method improves recommendation accuracy of state-of-the-art CF models for previous users and items with few ratings, as well as cold start users and items with no rating.
\end{abstract}

Keywords: Collaborative Filtering, Recommender Systems, Explanation, Cold Start, Tag-Keyword

\section{Introduction}

Collaborative filtering $(\mathrm{CF})$ has been proposed as a preferred technique for building recommender systems, which recommends items to users by information filtering on the past ratings of users on items. In the last few years, modelbased CF methods, such as latent factor models [1, 2, 3], have become not only a hotspot in academic research, but also a focus concerned by industrial community due to their good accuracy and efficiency on large and sparse dataset. However, pure model-based methods face some crucial challenges (e.g, sparsity, cold start, prediction quality, scalability, etc). Despite that two recent attempts: context-aware $[4,5,6,7]$ and social-aware $[8,9,10,11,12,13,14]$ have been conducted in the challenges, there are still two problems existing in most of the improved methods.

One important problem is explanation [15], which gets little attention of researchers in the past. The premise behind latent factor models is that user behaviour is determined by a small number of unobserved factors, and user's ratings on item are modeled by linearly combining user and item vectors of the factors. First, it is difficult to know what do the factors represent explicitly, which make it difficult for application providers to identify whether the recommended items match user interest. Second, making recommendation arbitrarily with the ratings is inefficient,

Email address: $13112081 @ b j t u . e d u . c n, ~ T e l .:+8618811445774(\mathrm{Ke} \mathrm{Ji})$ 


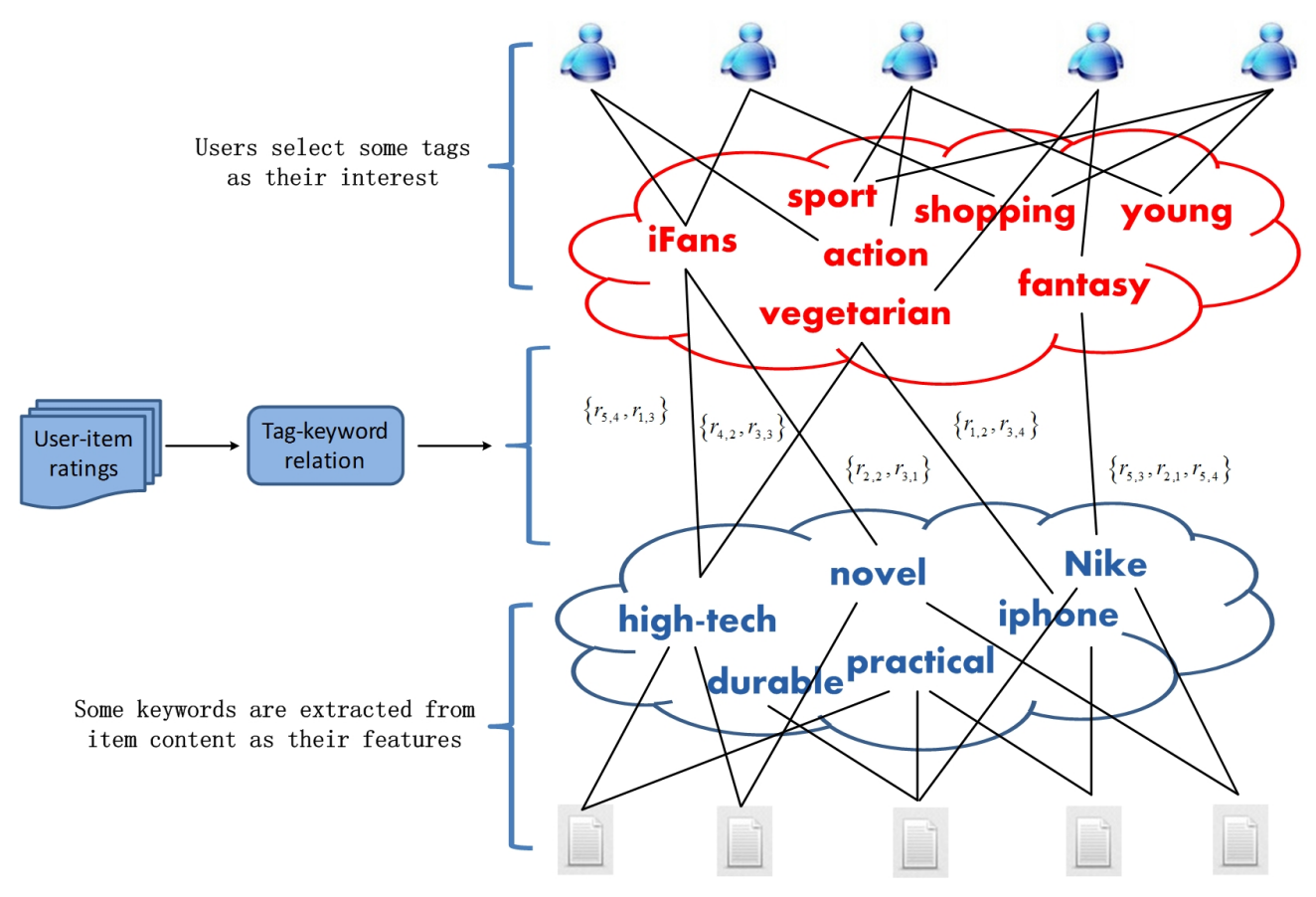

Figure 1. User-item ratings establish the relationships between users' tags and items' keywords.

which make it difficult for users to accept the recommended items. Many times, the recommended items are quite to match user interest, but most users have an idle nature, cannot be proactive in clicking the links to take a closer look at the strange items, which make users missing the useful items. So an excellent recommender system needs not only to provide the items users really want, but also to help users understand the recommended items.

Another important problem is cold start (i.e, new users and new items) [16], viewed as a special case of the sparsity that happens in most of CF methods. From application providers perspective, the users and items newly added to the system contain a potential for huge new transactions. Meanwhile new items may get the special attention of users. Hence, efficiency of cold start recommendation is very important to providers' profit growth and users' satisfaction. Latent factor models that rely on user's and item's ratings cannot make recommendations for new users and new items since no rating is available to form their latent factor vectors. Some recent methods $[17,18,11,10,19,20,21]$ are proposed to handle cold start users or items by incorporating additional information, such as content, context and social network. But no method is effective in the specific case of recommending new items to new users. Besides, in a practical application, the situations that context is difficult to get and social network is very sparse limit the use of the models. Therefore, cold start problem still needs further improvement.

Rather than complex context and social information, content-based information (i.e, user's tags and item's keywords) is not only more easily obtained, but also more direct to describe user interest and item features. User-item ratings establish the relationships between users' tags and items' keywords (Figure 1). A promising approach thus arises: we can build content association between user and item based on the tag-keyword relation (Figure 2), and then recommend the items with high similarity in content to users. This approach can not only give a good explanation of the recommendation results, but also use the previous tags and keywords to identify new users' interest and new items' features. However, there are three main challenges for designing this approach: (1) there are missing tag-keyword relations that is no related to user-item ratings; (2) every user has different interest in selected tags; (3) every item has different correlation with extracted keywords.

Based on the above discussion, we propose a hybrid method which generate explainable recommendation, meanwhile address cold start problem to some extent by incorporating content-based information and social information. We first propose a novel neighbor-based approach for helping to build the tag-keyword relation matrix, where partial missing entries are calculated based on the known relations between tags and keywords with the highest co-occurrence 


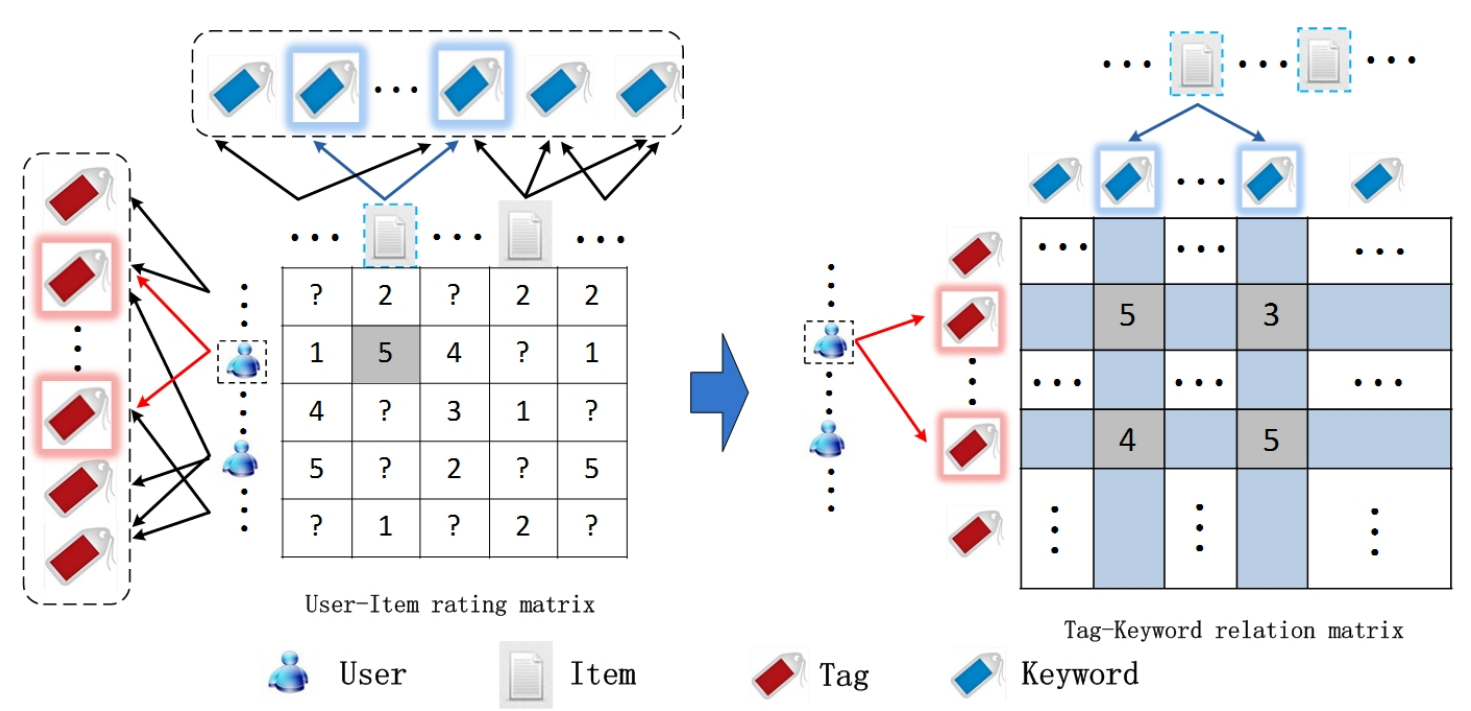

Figure 2. We can make prediction based on the tag-keyword relation instead of the user-item rating matrix. But, there are missing tag-keyword relations that is no related to user-item ratings; every user has different interest in selected tags; every item has different correlation with extracted keywords.

frequency. Then, we propose a novel 3-factor matrix factorization model over the rating matrix for learning every user's interest vector for selected tags and every item's correlation vector for extracted keywords. In particular, we introduce a social regularization term to help shape user interest vectors by considering the influence of a user's friends. Finally, interest vectors, correlation vectors and tag-keyword relation matrix are integrated to make recommendations. Analysis shows our method can generate explainable recommendation results with simple descriptions attached to every recommended item that help users understand the item, and extensive experiments on real data show the accuracy improvement of our method over state-of-the-art CF models on making recommendations for previous users and items with few ratings, as well as cold start users and items with no rating.

The rest of this paper is organized as follows: In Section 2, we review the related work. In Section 3, we give the problem formulation. In Section 4, we present our method in detail. In Section 5, we report the experimental settings and results. In Section 6, we conclude this paper.

\section{RELATED WORK}

In this section, we review several major approaches to recommender systems [22] (content-based filtering, collaborative filtering and latent factor model) and the problems (explanation and cold start).

\subsection{Content-based and Collaborative Filtering}

Content-based filtering analyzes a set of descriptions of items previously rated by a user, and maintain a list of features for the user. The basic idea is to recommend the items based on how well the features of items match up the features of the user. Recently, some topic model-based methods [23, 24, 21] have been proposed to make recommendation based on semantic content. Content-based filtering has several advantages that not only provide good explanation on why the items occur in the recommended list, but also have the ability of recommending cold start items not yet rated to any user; nevertheless, the working on individual user needs specialized content-based technique and enough user ratings, which limit the application range of this method.

Collaborative filtering [25] relies on user-item ratings, which mainly contains two classes: memory-based and model-based. Memory-based [26, 27, 28, 29] use similarity comparison (e.g., VSS or PCC [27]) to build neighborhood relationship between users or items, and predict missing ratings based a weighted sum of ratings from similar users or items. Model-based [1, 2, 3], in contrast, use user-item ratings to train a model first, and then make recommendation via the model. Many experimental results and conclusions argue that model-based methods lead to somewhat more accurate results on large and sparse data, while memory-based methods have some practical advantages. 


\subsection{Latent Factor Model}

Latent factor model, such as matrix factorization is a popular model-based approach, which decomposes $m \times n$ rating matrix $R$ into two low-rank matrices: $U \in R^{m \times d}$ and $V \in R^{n \times d}(d<\min (m, n))$, the product of which is used to construct a matrix approximation $R \approx U V^{T}$. One of the most popular methods by minimizing the sum-of-squarederrors objective function with quadratic regularization terms is

$$
\mathcal{L}=\sum_{u=1}^{m} \sum_{v=1}^{n} I_{u, v}\left(R_{u, v}-U_{u} V_{v}^{T}\right)^{2}+\lambda_{1}\|U\|_{F}^{2}+\lambda_{2}\|V\|_{F}^{2}
$$

where $\lambda_{1}$ and $\lambda_{2}$ is the extent of regularization and $\|\cdot\|_{F r o}$ is the Frobenius norm. $I_{u, v}$ is the indicator function that is equal to 1 if user $u$ rated item $v$ and equal to 0 otherwise. The optimization problem in Equation 1 can be solved by performing gradient decent on $U_{u}$ and $V_{v}$. Then, the missing rating can be predicted by $U_{u} V_{v}^{T}$.

Pure model-based methods have several challenges, such as data sparsity, scalability, cold start and poor prediction. Two recent attempts have been done to alleviate the above problems, one with contextual information $[6,7]$ and one with social information $[12,13,14]$. Context-aware recommendation $[4,5]$ take additional specific contexts (time, location emotion, review, etc) into account to give more accurate prediction. Social recommendation $[8,9,10,11]$ incorporating social networks information improve prediction quality and cold start user.

\subsection{Explanation and Cold Start}

In recommender systems, the accuracy metrics, such as MAE and RMSE are often used to evaluate the recommendation quality. However, the good prediction accuracy on benchmark datasets do not mean that we can make maximum use of recommender systems. In real world applications, lack of explanation may greatly reduce efficiency of the recommendation results. For example, poor explanation on how the system works reduces the reliability of recommender systems [30], and poor explanation on the recommended items reduces the acceptance of user on the items [31]. There is not much previous work on explanation [32, 33].[32] proposed a unied framework, Personalized Semantic Ranking (PSR), to incorporate the user-generated content (UGC), and utilize the semantic information extracted from UGC to serve as proper explanations for recommendation. [33] proposed a Social-Relational Topic Model (SRTM), which jointly models UGC and social links into a unified generative process for alleviating the effect of topic-irrelevant links and obtaining reliable topics to explain the recommendation. But, they ignore the itemgenerated content, and cannot give the clear answer which item feature most attract the current user's interest to the given item.

Cold start is a common problem in recommender systems. Although latent factor model improves prediction accuracy when rating data is sparse, it does not work for cold-start recommendation where no rating data on users or items is available. There are some recent hybrid approaches on cold start: some with content information [17, 18], some with social information [11, 10] and some with context information [20,19,34]. But the problem is far from solved completely, especially recommending new items to new users.Recently, some cross-platform based methods $[35,36]$ are proposed to alleviating the data sparsity and cold-start problems. Based on the knowledge on different platforms, they explore the cross-platform collaborative relationship derived from content-based and network-based information to improve the recommendation. But, as to user privacy protection and industrial competition, the other platforms are often governed strictly on access for the related knowledge, which limit their scope of use.

In recent years, tag recommendation [37] and keyword extraction [38] techniques are largely applicable to the web. Tag and keyword both provide meaningful content descriptors of the objects, where tags selected by users have expressions of their interest and keywords extracted from items mean their features. That motivates us to attempt to improve explanation and cold start problems by combining content relationship between user and item with user-item ratings for the recommendation task.

\section{PROBLEM FORMULATION}

Suppose that we have a set of users $\mathcal{U}=\left\{u_{1}, \cdots, u_{m}\right\}$ and a set of items $\mathcal{V}=\left\{v_{1}, \cdots, v_{n}\right\}$, and the ratings are expressed by a matrix $R \in R^{m \times n}$. Let $\mathcal{K}_{U}=\left\{k_{1}^{U}, \cdots, k_{M}^{U}\right\}$ be a set of tags and $\mathcal{K}_{V}=\left\{k_{1}^{V}, \cdots, k_{N}^{V}\right\}$ be a set of keywords. $K_{U}(u)$ denotes the tags selected by user $u$ and $K_{V}(v)$ denotes the keywords extracted from item $v$ (an example is shown 


\begin{tabular}{c|c} 
User & Tags \\
\hline$u_{1}$ & $\{$ sport, iFans,action\} \\
$u_{2}$ & $\{$ iFans, shopping \\
$u_{3}$ & $\{$ sport, action, young \\
$u_{4}$ & $\{$ vegetarian, fantasy\} \\
$u_{5}$ & \{sport, shopping, young
\end{tabular}

(a) Users' tags

\begin{tabular}{c|c} 
Item & Keywords \\
\hline$v_{1}$ & \{durable, practical\} \\
$v_{2}$ & $\{$ iphone, high-tech, novel\} \\
$v_{3}$ & $\{$ Nike, durable, practical\} \\
$v_{4}$ & \{practical, high-tech, durable\} \\
$v_{5}$ & \{Nike, novel, durable\}
\end{tabular}

(b) Items' keywords

Figure 3. Example users' tags and items' keywords datasets

in Figure 3). We define the tag-keyword relation matrix $S \in R^{M \times N}$, where $S_{i, j}$ denotes the similarity between tag $i$ and keyword $j$. To consider user's different interest in selected tags, we define an interest vector $P_{u}=\left(p_{u}^{(1)}, \cdots, p_{u}^{\left|K_{U}(u)\right|}\right)$ for every user, the $z$-th element $p_{u}^{(z)}$ of which defines user $u$ 's interest in the $z$-th tag of $K_{U}(u)$. Likewise, to consider item's different correlation with extracted keywords, we define correlation vector $Q_{u}=\left(q_{v}^{(1)}, \cdots, q_{v}^{\left|K_{V}(v)\right|}\right)$ for every item, the $z$-th element $q_{v}^{(z)}$ of which defines item $v$ 's correlation with the $z$-th keyword of $K_{v}(v)$.

Our goal is to build content association between user and item based on the three factors-tag-keyword relation matrix $S$, user interest vector $P_{u}$ and item correlation vector $Q_{u}$, and then recommend the items with high similarity in content to users.

\section{OUR METHOD}

As mentioned before, our method relies on having: (1) tag-keyword relation matrix $S$; (2) user interest vector $P_{u}$ for selected tags; (3) item correlation vector $Q_{v}$ for extracted keywords. In this section, we will systematically describe our solutions for parameters estimation and prediction.

\subsection{Building the Tag-Keyword Relation Matrix}

User-item ratings establish the relationships between users' tags and items' keywords. Let the tuple $\left\langle k_{i}^{U}, k_{j}^{V}, R_{z^{*}}\right\rangle$ denote a set of ratings $R_{z^{*}}$, where $z^{*}$ is a user-item pair $(u, v)$ that contain tag $k_{i}^{U}$ and keyword $k_{j}^{V}$ respectively. We first initialize partial entries of the relation matrix $S$ that is related to the observed tuples. Then, we exploit the known entries for filling in the missing entries.

Initialization. Generally, a tag is selected by multiple users and a keyword is shared by multiple items, leading to multiple instances of the same tag-keyword pair with different rating values. Assume that we have a list of tuples related to tag $i$ and keyword $j$

$$
\Phi_{i, j}=\left\{\left\langle k_{i}^{U}, k_{j}^{V}, R_{1^{*}}\right\rangle,\left\langle k_{i}^{U}, k_{j}^{V}, R_{2^{*}}\right\rangle, \cdots,\left\langle k_{i}^{U}, k_{j}^{V}, R_{N^{*}}\right\rangle\right\}
$$

and $\vec{n}_{i, j}=\left\{n_{i, j}^{(1)}, \cdots, n_{i, j}^{(r)}\right\}$ count the occurrences of rating scale $\{1, \cdots, r\}$ with $N^{*}$ total ratings. We then compute $S_{i, j}$ as

$$
S_{i, j}=\sum_{z=1}^{r} \frac{n_{i, j}^{(z)} z}{N^{*}}
$$

which ensures $S_{i, j}$ is a weighted sum of the ratings in $\Phi_{i, j}$. A simple example of the calculation is shown in Figure 4(a).

Fill in the missing entries. This task can be done by any typical CF approach, such as memory-based or modelbased methods that suffer from the sparsity of the rating matrix. However, what it different from general case about the rating matrix is that some tags or keywords frequently appear together in users' $K_{U}(u)$ or items' $K_{V}(v)$ (e.g, 
The ratings in the tuples $\Phi_{i, j}$

\begin{tabular}{c|c|c|} 
User & Item & Rating \\
\cline { 1 - 2 }$u_{2}$ & $v_{1}$ & 5 \\
$u_{3}$ & $v_{2}$ & 3 \\
$u_{1}$ & $v_{3}$ & 5
\end{tabular}$\vec{n}_{i, j}=(0,0,1,1,3)$

(a) Initializing one entry

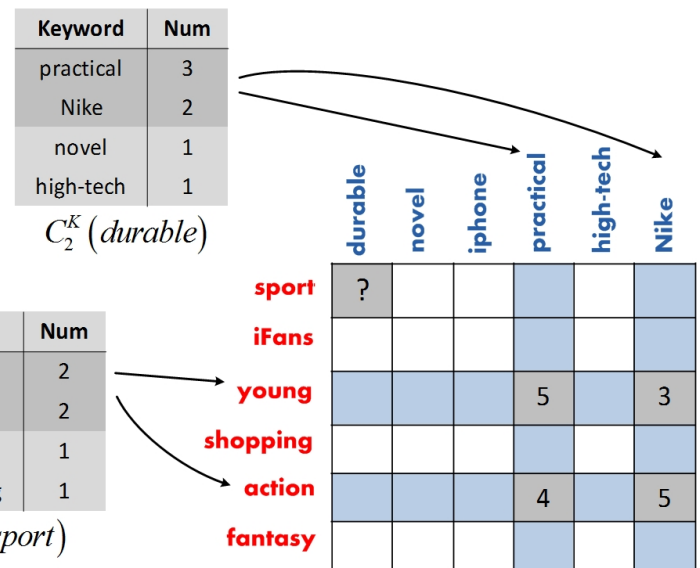

$S_{\text {sport, durable }}=\frac{1}{18}[(2+3) \times 5+(2+2) \times 3+(2+3) \times 4+(2+2) \times 5] \approx 4.3$

(b) Filling one missing entry

Figure 4. A example of initializing one entry $S_{i, j}$ of the relation matrix based on the ratings $\left\{R_{1^{*}}, R_{2^{*}}, \cdots, R_{N^{*}}\right\}$ in the tuples $\Phi_{i, j}$, and a example

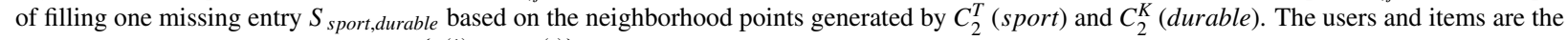
examples in Figure 3. Note that $\vec{n}_{i, j}=\left\{n_{i, j}^{(1)}, \cdots, n_{i, j}^{(r)}\right\}$ count the occurrences of rating scale $\{1, \cdots, r\}$ with $N^{*}$ total ratings.

tags \{sport, yong\} and keywords \{durable, practical $\}$ in Figure 3). We therefore propose a neighbor-based approach for calculating partial missing entries based on the known relations between tags and keywords with the highest co-occurrence frequency, meaning a preprocessing for improving CF approach against the sparsity of $S$. Let $C_{k}^{T}(i)$ denote a set of tags that have Top - $k$ co-occurrence frequency with tag $i$ and $C_{k}^{K}(j)$ denote a set of keywords that have Top $-k$ co-occurrence frequency with keyword $j$. We compute the missing $S_{i, j}$ using a weighted sum of the neighbor entries related to $C_{k}^{T}(i)$ and $C_{k}^{K}(j)$.

$$
S_{i, j}=\sum_{z \in C_{k}^{T}(i)} \sum_{w \in C_{k}^{K}(j)} \frac{N_{i, z}^{T}+N_{j, w}^{K}}{N(i, j)} I_{z, w} S_{z, w}
$$

where $N_{i, z}^{T}$ denote the co-occurrence number of tags $i$ and $z$, and $N_{j, w}^{K}$ denote the co-occurrence number of keywords $j$ and $w . N(i, j)$ is the normalization

$$
N(i, j)=\sum_{z \in C_{k}^{T}(i)} \sum_{w \in C_{k}^{K}(j)} I_{z, w}\left(N_{i, z}^{T}+N_{j, w}^{K}\right)
$$

where $I_{z, w}$ is the indicator function that is equal to 1 if $S_{z, w}$ is known and equal to 0 otherwise, ensuring that $S_{i, j}$ only weights across the known $S_{z, w}$. A simple example of calculating $S_{i, j}$ using the $T o p-2 C_{2}^{T}(i)$ and $C_{2}^{K}(j)$ is shown in Figure 4(b). After the preprocessing stage, we apply a CF approach on $S$ to fill in all the rest missing entries. Note that our preprocessing, at best, can fill in all the missing entries, which eliminates the need to apply CF approach; at worst, do not fill in any entry, which maintains CF approach on the original $S$.

\subsection{Learning User Interest vector and Item Correlation Vector}

Although we build the full relation matrix $S$, a user has different interest in selected tags and an item have different correlation with extracted keywords. In order to provide personalized and accurate recommendation, we propose a 3factor matrix factorization model for learning interest vector $P_{u}$ and correlation vector $Q_{v}$. Let $S_{u, v}^{\prime}$ denote a sub-matrix of $S$ only corresponding to the relations between user $u$ 's tags $K_{U}(u)$ and item $v$ 's keywords $K_{V}(v)$. 
We define the prediction $\hat{R}_{u, v}$ of user $u^{\prime}$ rating on item $v$ as a bilinear function of $P_{u}$ and $Q_{v}$ in the following:

$$
\hat{R}_{u, v}=\sum_{a=1}^{\left|K_{U}(u)\right|\left|K_{V}(v)\right|} \sum_{b=1} P_{u, a}\left(S_{u, v}^{\prime}\right)_{a, b} Q_{v, b}
$$

where the known $\left(S_{u, v}^{\prime}\right)_{a, b}$ measures the affinity of the two factors $P_{u, a}$ and $Q_{v, b}$. The equation is rewritten as a simple form (an example is shown in Figure 5):

$$
\hat{R}_{u, v}=P_{u} S_{u, v}^{\prime} Q_{v}^{T}
$$

Furthermore, we formulate the optimization problem for finding the best $P_{u}$ and $Q_{v}$ as

$$
\mathcal{L}=\sum_{u=1}^{m} \sum_{v=1}^{n} I_{u, v}\left(R_{u, v}-P_{u} S_{u, v}^{\prime} Q_{v}^{T}\right)^{2}+\frac{\lambda_{1}}{2} \sum_{u=1}^{m}\left\|P_{u}\right\|_{F}^{2}+\frac{\lambda_{2}}{2} \sum_{v=1}^{n}\left\|Q_{v}\right\|_{F}^{2}
$$

which transforms traditional 2-factor factorization in Equation 1 into a 3-factor factorization in Equation 7. The advantage of our method is that the dimensions of the vectors $P_{u}$ and $Q_{v}$ are equal to the number of $K_{U}(u)$ and $K_{V}(v)$, which relax the low-rank assumption in traditional matrix factorization. A local minimum of the objective function can be found by performing gradient descent on $P_{u}$ and $Q_{v}$ for all users and items.

$$
\begin{gathered}
\frac{\partial \mathcal{L}}{\partial P_{u}}=\sum_{v=1}^{n} I_{u, v}\left(P_{u} S_{u, v}^{\prime} Q_{v}^{T}-R_{u, v}\right) Q_{v}\left(S_{u, v}^{\prime}\right)^{T}+\lambda_{1} P_{u} \\
\frac{\partial \mathcal{L}}{\partial Q_{v}}=\sum_{u=1}^{m} I_{u, v}\left(P_{u} S_{u, v}^{\prime} Q_{v}^{T}-R_{u, v}\right) P_{u} S_{u, v}^{\prime}+\lambda_{2} Q_{v}
\end{gathered}
$$

\subsection{An Enhanced Model by Social Regularization}

On the basic of our 3-factor matrix factorization model presented in the previous section, we propose an enhanced model taking into account social information to better shape user interest. Inspired by SoReg [11], we make an assumption: if user $i$ has a friend $f$, there is a similarity between their interest. But, unlike user latent factor vectors with the same dimension in SoReg, the dimensions of user interest vectors in our method are equal to the number of every user's tags, different from each other. Hence, we cannot use SoReg directly to impose constraints on user interest vectors. Even so, every user's interest on all keywords can be calculated as $P_{u} S_{u}^{\prime}$, where $S_{u}^{\prime}$ is a sub-matrix of $S$ corresponding to the relations between user $u$ 's tags $K_{U}(u)$ and all keywords $\mathcal{K}_{V}$. All users' $P_{u} S_{u}^{\prime}$ are in the same dimension (an example is shown in Figure 6), and we propose a social regularization term

$$
\frac{\alpha}{2} \sum_{u=1}^{m} \sum_{f=\mathcal{F}(u)} \operatorname{sim}(u, f)\left\|P_{u} S_{u}^{\prime}-P_{f} S_{f}^{\prime}\right\|_{F}^{2}
$$

to constrain interest on all keywords between one user and their friends, where $\alpha$ is a constant controlling the extent of social regularization and $\operatorname{sim}(u, f)$ is the similarity function. In this paper, we apply Pearson Correlation Coefficient $(P C C)$ to measure the similarity.

$$
\operatorname{sim}(u, f)=\frac{\sum_{j \in I(u) \cap I(f)}\left(R_{u, j}-\bar{R}_{u}\right)\left(R_{f, j}-\bar{R}_{f}\right)}{\sqrt{\sum_{j \in I(u) \cap I(f)}\left(R_{u, j}-\bar{R}_{u}\right)^{2}} \sqrt{\sum_{j \in I(u) \cap I(f)}\left(R_{f, j}-\bar{R}_{f}\right)^{2}}}
$$

With the social regularization, our model is redefined as

$$
\mathcal{L}=\sum_{u=1}^{m} \sum_{v=1}^{n} I_{u, v}\left(R_{u, v}-P_{u} S_{u, v}^{\prime} Q_{v}^{T}\right)^{2}+\frac{\alpha}{2} \sum_{u=1}^{m} \sum_{f=\mathcal{F}^{+}(u)} \operatorname{sim}(u, f)\left\|P_{u} S_{u}^{\prime}-P_{f} S_{f}^{\prime}\right\|_{F}^{2}+\frac{\lambda_{1}}{2} \sum_{u=1}^{m}\left\|P_{u}\right\|_{F}^{2}+\frac{\lambda_{2}}{2} \sum_{v=1}^{n}\left\|Q_{v}\right\|_{F}^{2}
$$




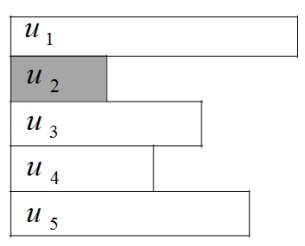

User interest vectors

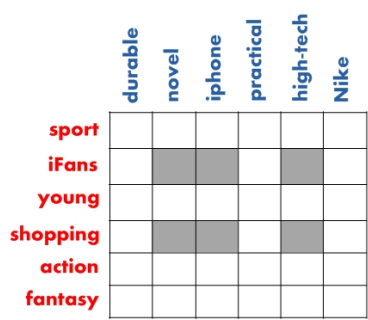

Tag-keyword relation matrix
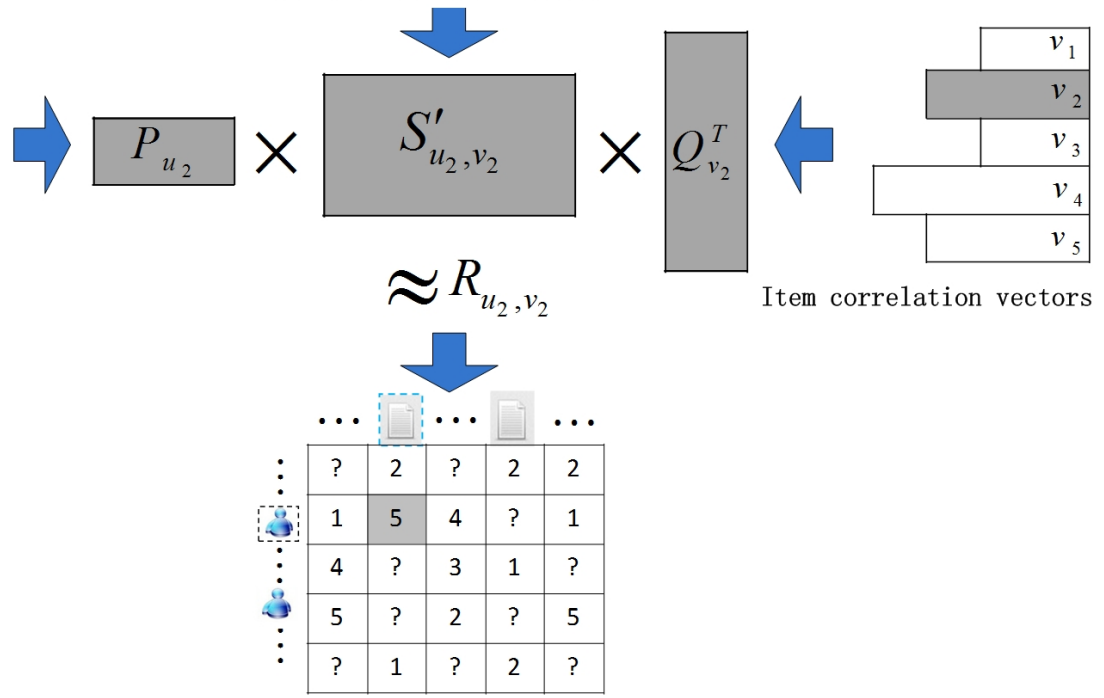

Item correlation vectors

User-Item rating matrix

Figure 5. With an extra factor: sub-relation matrix $S_{u, v}^{\prime}$, we transform the traditional 2-factor matrix factorization $U_{u} V_{v}^{T}$ in Equation 1 into a 3-factor matrix factorization $P_{u} S_{u, v}^{\prime} Q_{v}^{T}$ in Equation 7.

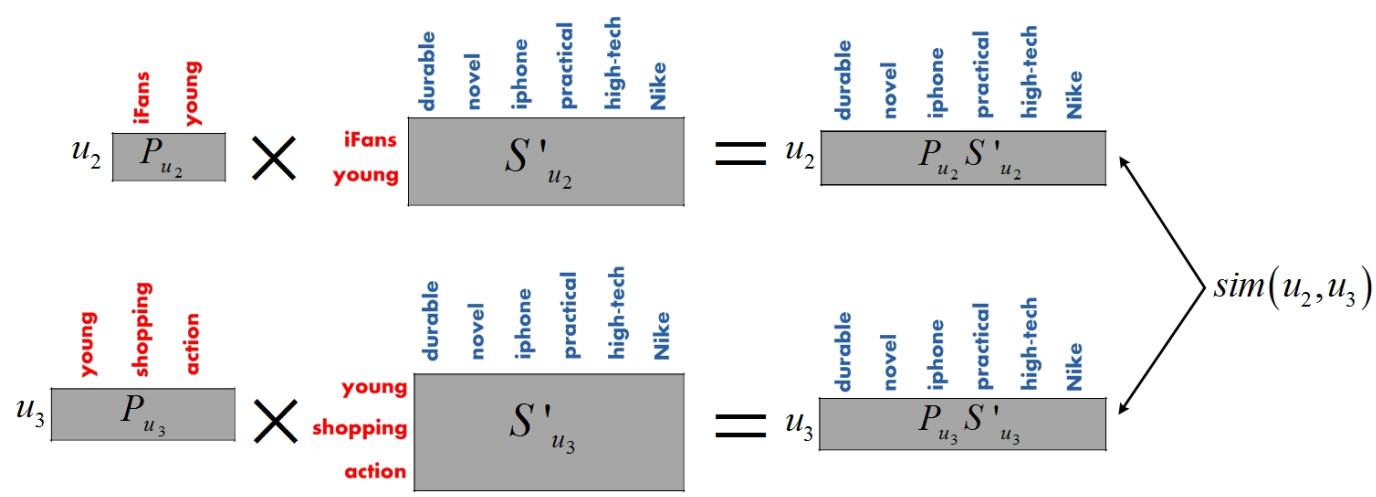

Figure 6. $P_{u} S_{u}^{\prime}$ with the same dimension represents every user $u$ 's interest on all keywords $\mathcal{K}_{V}$. Based on the concept of "social recommendation" that users may share similar interest with their friends, there is similar interest on all keywords between one user and their friends. 
The new gradient descents on $P_{u}$ and $Q_{v}$ are

$$
\begin{gathered}
\frac{\partial \mathcal{L}}{\partial P_{u}}=\sum_{v=1}^{n} I_{u, v}\left(P_{u} S_{u, v}^{\prime} Q_{v}^{T}-R_{u, v}\right) Q_{v}\left(S_{u, v}^{\prime}\right)^{T}+\sum_{f \in \mathcal{F}^{+}(u)} \operatorname{sim}(u, f)\left(P_{u} S_{u}^{\prime}-P_{f} S_{f}^{\prime}\right)\left(S_{u}^{\prime}\right)^{T} \\
+\sum_{g \in \mathcal{F}^{-}(u)} \operatorname{sim}(u, g)\left(P_{u} S_{u}^{\prime}-P_{g} S_{g}^{\prime}\right)\left(S_{u}^{\prime}\right)^{T}+\lambda_{1} P_{u} \\
\frac{\partial \mathcal{L}}{\partial Q_{v}}=\sum_{u=1}^{m} I_{u, v}\left(P_{u} S_{u, v}^{\prime} Q_{v}^{T}-R_{u, v}\right) P_{u} S_{u, v}^{\prime}+\lambda_{2} Q_{v}
\end{gathered}
$$

where $\mathcal{F}^{+}(u)$ denote user $u$ 's outlink friends and $\mathcal{F}^{-}(u)$ denote user $u$ 's inlink friends.

\subsection{Training and Prediction}

Our method consists of the following two steps: on the first step, we propose a novel neighbor-based approach for building the relation matrix $S$; on the second step, we propose a novel 3-factor matrix factorization model for learning interest vector $P_{u}$ and correlation vector $Q_{v}$. The whole training algorithm is shown in Algorithm 1.

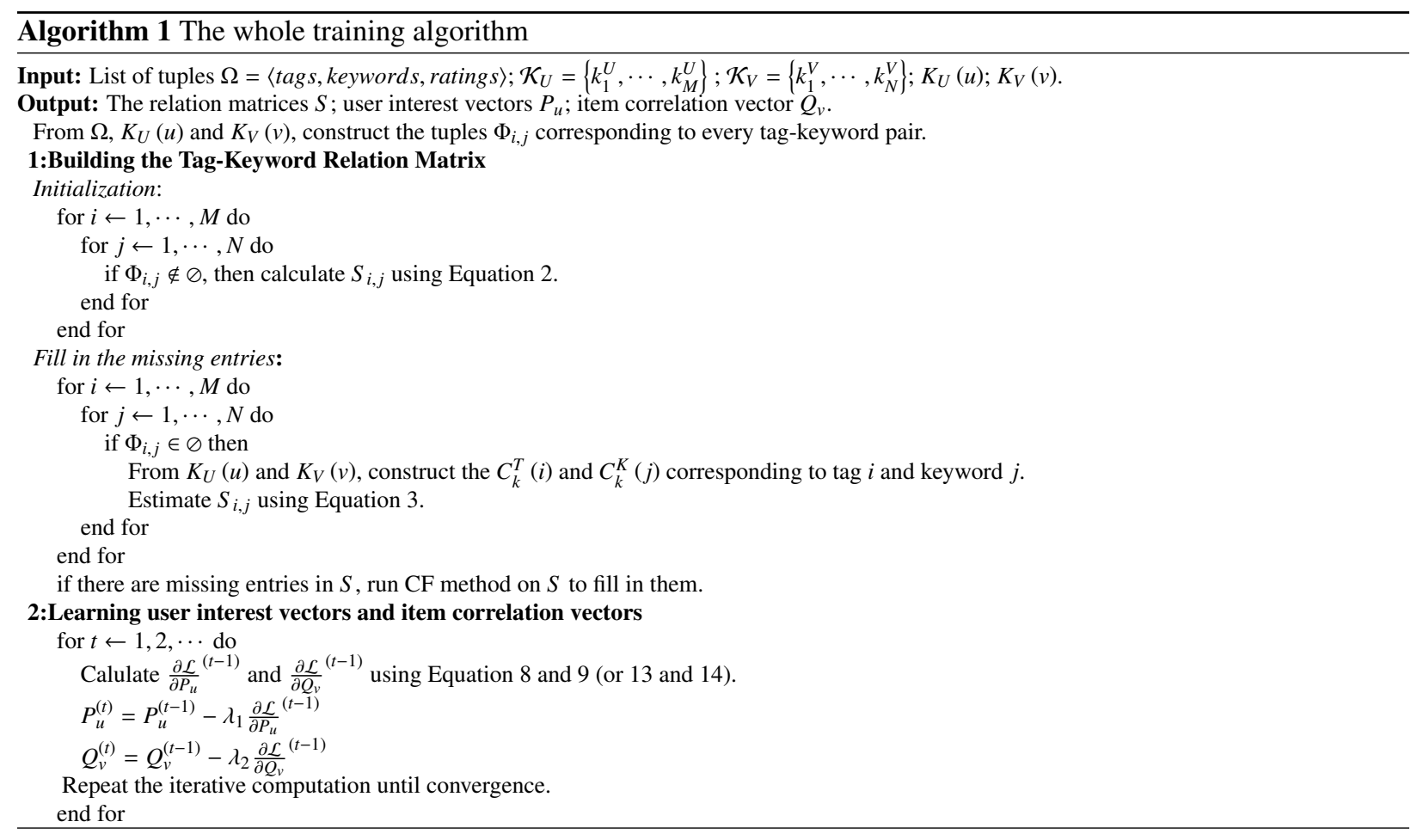

Explainable recommendation: Given a user-item pair $(u, v), P_{u} S_{u, v}^{\prime}$ is a $1 \times\left|K_{V}(v)\right|$ vector whose elements represent user $u$ 's interest in item $v$ 's keywords. By integrating with $Q_{v}$ correlation on every keyword, we have a set

$$
\Upsilon(u, v)=\left\{\left[P_{u} S_{u, v}^{\prime}\right]_{1} Q_{v, 1},\left[P_{u} S_{u, v}^{\prime}\right]_{2} Q_{v, 2}, \cdots\right\}
$$

which correspond with user interest on item $v$ 's keywords. Note that the sum of elements in $\Upsilon(u, v)$ is the prediction (i.e, $P_{u} S_{u, v}^{\prime} Q_{v}^{T}$ ) for user $u$ 's rating on item $v$. Based on the values of $\Upsilon(u, v)$, we can select some of the best keywords from $K_{V}(v)$ to design a simple description attached to the recommended item. For instance, for a "sport" user, the keywords "practical" and "durable" may draw more user's attention rather than other keywords in $K_{V}(v)$ do. So we take the two keywords as an identifier to help recommending the shoes to the "sport" user, even can form a message prompt 
The Nike shoes are practical and durable, which you may need right now.

Obviously, users prefer to the products understood intuitively rather than the strange products lacking of description information. And, compared to previous work [32,33], the method can explicitly declare which item feature may attract the current user's interest.

Cold start recommendation: In addition to making recommendations for present users and items, our method can perform the following cold start recommendation for new users and new items:

- New user. If the new user $u_{n e w}$ 's tags are known and included in $\mathcal{K}_{U}$, we can compute user $u_{\text {new }}$ 's rating on any item $v$ using $P_{u_{\text {new }}} S_{u_{\text {new }},}^{\prime} Q_{v}^{T}$. However, we cannot learn $P_{u_{\text {new }}}$ because there is no user $u_{\text {new }}$ 's historical rating data. In this case, every tag in $\mathcal{K}_{U}\left(u_{\text {new }}\right)$ is treated fairly:

$$
P_{u_{\text {new }}}=\left(\frac{1}{\left|\mathcal{K}_{U}\left(u_{\text {new }}\right)\right|}, \cdots, \frac{1}{\mathcal{K}_{U}\left(u_{\text {new }}\right)}\right)
$$

- New item. If the new item $v_{n e w}$ 's keywords are known and included in $\mathcal{K}_{V}$, we can compute any user $u$ 's rating on item $v_{\text {new }}$ using $P_{u} S_{u, v_{\text {new }}}^{\prime} Q_{v_{\text {new }}}^{T}$. However, we cannot learn $Q_{v_{\text {new }}}$ because there is no item $v_{\text {new }}$ 's historical rating data. In this case, every keyword in $\mathcal{K}_{V}\left(v_{\text {new }}\right)$ is treated fairly:

$$
Q_{v_{\text {new }}}=\left(\frac{1}{\left|\mathcal{K}_{V}\left(v_{\text {new }}\right)\right|}, \cdots, \frac{1}{\mathcal{K}_{V}\left(v_{\text {new }}\right)}\right)
$$

- New users and new items. In this extreme case, our model can still make recommendations as long as the new user $u_{\text {new }}$ 's tags and new item $v_{\text {new }}$ 's keywords are known. The prediction formula is $P_{u_{\text {new }}} S_{u_{\text {new }}, v_{\text {new }}}^{\prime} Q_{v_{\text {new }}}^{T}$, where $P_{u_{\text {new }}}$ and $Q_{v_{\text {new }}}$ are Equation 16 and 17.

\section{EXPERIMENTS}

\subsection{Datasets}

Tencent Weibo ${ }^{1}$ is one of the largest Chinese online social websites. As a micro-blogging, it has become a major online communication platform for following some items' trend, building friendship and sharing interest. In our experiments, we choose the real Tencent Weibo data published by KDD Cup $2012^{2}$. This dataset is much bigger and richer than before (Netflix, Movielens, Epinions and so on), sampling from 425 million weibo users for 50 days and containing 2 million active users, more than 6000 items, 300 million recommendation records and 3 million following actions. Meanwhile, the dataset contains social network information and much description information such as user's tags, item's keywords, item's category, timestamps and so on. We will predict user's action to items, where 1 represents the user accepts the recommendation of item, and 0 represents the user rejects the recommended item.

We extract a small dataset over a period of time randomly. The basic statistics of the dataset are summarized in Figure 7 (a). The density of the user-item rating matrix is

$$
\frac{379598}{12518 \times 3610}=0.83 \%
$$

The average user has 30 following/not following actions. We divide the rating data into five parts (Figure 7 (b)). The biggest part is used as the training set $R_{\text {train }}$ and the test set $R_{\text {test }}$. Only the users of dataset $R_{\text {new }}^{U}$ do not exist in the training data. Only the items of dataset $R_{\text {new }}^{I}$ do not exist in the training data. Both the users and items of dataset $R_{\text {new }}^{U I}$ do not exist in the training data. Different proportions of $R_{\text {train }}$ and $R_{\text {test }}$ will be applied to the performance comparison experiments. We use $R_{\text {new }}^{U}, R_{\text {new }}^{I}$ and $R_{\text {new }}^{U I}$ to test the performance of our model for cold start recommendation in Section 5.6.

\footnotetext{
${ }^{1}$ http://t. qq. com/

${ }^{2}$ http://www. kddcup2012. org/
} 


\begin{tabular}{|c|c|}
\hline Description & Value \\
\hline User number & 12518 \\
\hline Item number & 3610 \\
\hline Rating number & 375989 \\
\hline Tag number & 13250 \\
\hline Keyword number & 11021 \\
\hline Link number & 3898 \\
\hline
\end{tabular}

(a) The basic statistics

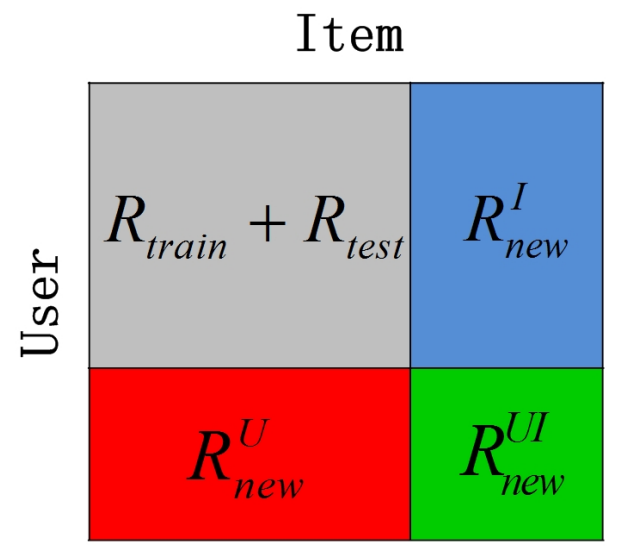

(b) The partition

Figure 7. The extracted dataset

\subsection{Metrics}

In our experiments, we use two kinds of popular metrics to evaluate the performance: one with rating, MAE; one with ranking, Recall.

$M A E$, short for Mean Absolute Error, is defined as

$$
M A E=\frac{1}{\left|R_{t e s t}\right|} \sum_{R_{u, v} \in R_{\text {test }}}\left|R_{u, v}-\hat{R}_{u, v}\right|
$$

where $R_{u, v}$ in $R_{\text {test }}$ denotes the information adoption value given to item $v$ by user $u$.The smaller MAE value means better performance.

Recall is defined as the ratio of accepted items $N_{r s}$ to total number of relevant items $N_{r}$

$$
\text { Recall }=\frac{N_{r s}}{N_{r}}
$$

The bigger Recall value means better performance.

\subsection{Comparisons and Implementation}

In order to show the performance improvement, we compare our method with traditional memory-based algorithms, matrix factorization, social recommendation and other state-of-the-art methods.

- Item-based CF algorithm [28] finds the relationships between items and then predicts the rating for the target item based on the weighted averaging of the ratings which have been rated by the target user.

- $\boldsymbol{P M F}$ is a matrix factorization model, proposed by Salakhutdinov et al. in [2], which outperforms traditional memory-based algorithms on the large, sparse and imbalanced rating matrix. Given the rank, it can build a matrix approximation which minimize the sum-of-squared instance to the rating matrix.

- SocialMF is a model-based approach in social networks, proposed by Jamali et al. in [10], which incorporates the mechanism of trust propagation. It makes the features of every user dependent on the feature vectors of the his direct neighbors. The model can improve accuracy for cold start users.

- SoReg is a matrix factorization model with social regularization, proposed by Ma et al. in [11]. It makes a distinction between "social friendships" and "trust relationships. In order to represent the various social friendships, the model treats dissimilar tastes of friends in the different social regularization. 

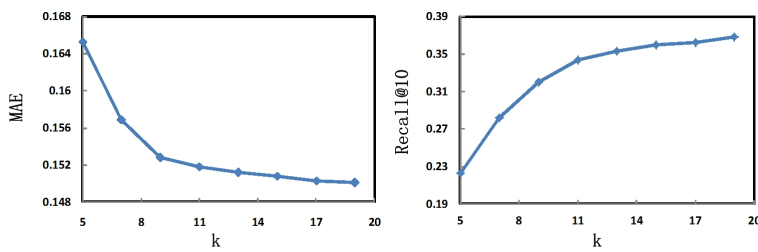

(a) MAE and Recall vs Neighbor Number
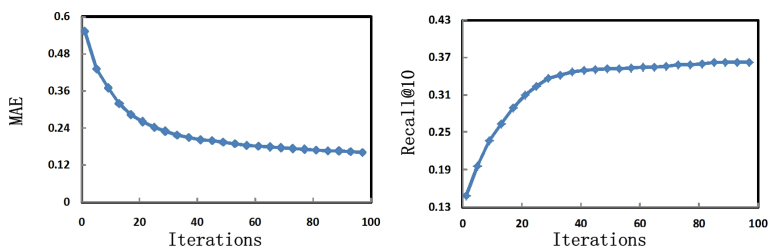

(b) MAE and Recall vs Iteration Number

Figure 8. Impact of parameters (Neighbor Number and Iteration Number)

- $\boldsymbol{L L R}$ is a two-phase layered learning framework, proposed by Ji et al. in [17], which incorporates the category, item content and social networks information. It can utilize the item's content similarity in the same category to overcome the difficulties of large amount and dynamic update of items.

We call our method proposed in Section $4 \boldsymbol{C S R}$. Meanwhile, we implement different configurations of our method to demonstrate the effectiveness of our neighbor-based approach and social regularization.

- $\boldsymbol{C S R} \boldsymbol{R}_{C F}^{S}$. This method uses Item-based to fill in $S$ without our preprocessing, and uses 3-factor matrix factorization model with social regularization in Equation 12.

- $\boldsymbol{C S R}_{M F}^{S}$. This method uses $P M F$ to fill in $S$ without our preprocessing, and uses 3-factor matrix factorization model with social regularization in Equation 12.

- $\boldsymbol{C S R}_{M F^{+}}^{-S}$. This method adds our preprocessing to $P M F$, and uses our basic 3-factor matrix factorization model in Equation 7.

- $\boldsymbol{C S R}_{M F^{+}}^{S}$. This method adds our preprocessing to $P M F$, and uses 3-factor matrix factorization model with social regularization in Equation 12.

If no indicator is specifically stated, $\boldsymbol{C S R}$ means $\boldsymbol{C S R}_{M F^{+}}^{S}$.

JAMA $^{3}$ is an open matrix package for Java, developed at NIST and the University of Maryland. It provides the fundamental operations of numerical linear algebra, such as matrix addition and multiplication, matrix norms and selected element-by-element array operations, etc. All models in our experiments are implemented using this library.

\subsection{Parameter Settings}

In this section, we analyze how the changes of the parameters affect the performance of our method. We aim to find the best settings by applying different parameter configurations.

Number of neighbors: On the first step, we use the neighbor entries related to tags and keywords with Top $-k$ co-occurrence frequency to fill in the missing entries. If $k$ is too small, the neighbor entries will be too unique, and there may even be no observed neighbor entries for the missing entry. If $k$ is too big, the complexity of searching neighbor entries and calculating weight coefficient will considerably increase. We conduct experiments with $k$ from 5 to 20. Figure 8(a) shows that with $k$ increasing, $M A E$ decreases and Recall increases reduce gradually, but when $k$ goes over 10, the values change rather slow. So we choose Top - 10 nearest neighbors.

Number of iterations: On the second step, the gradient descent method based on iteration computation is timeconsuming because each iteration needs to calculate gradient and update parameters. Too many times of iterations cannot necessarily get good results, and might even appear overfitting problem with high probability. We conduct experiments on different iterations. In Figure 8(b), we observe that $M A E$ decreases and Recall increases gradually with the number of iterations increasing. It is better to run 60 iterations because more than 60 iterations incur higher computational overhead without the big performance improvement in return. 


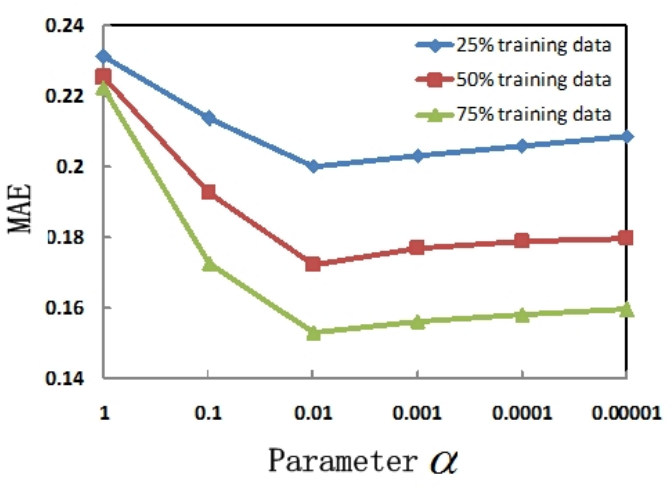

(a) MAE

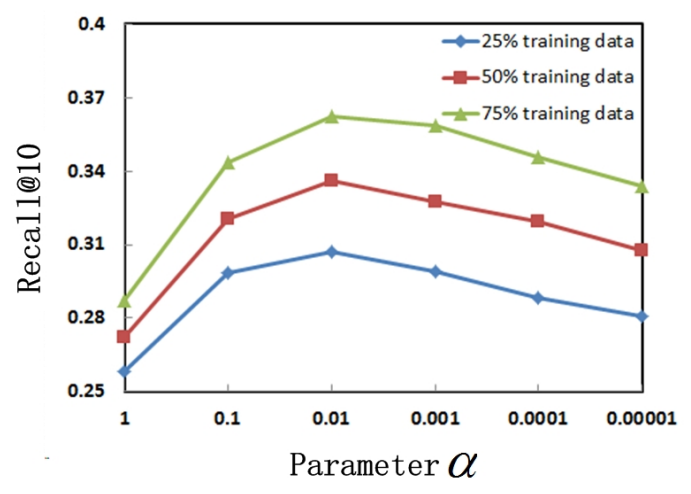

(b) Recall

Figure 9. Impact of Parameter $\alpha$

\subsection{Impact of Parameter $\alpha$}

In our 3 -factor matrix factorization model, the parameter $\alpha$ controls the influence of social information on the objective function (Equation 12), balancing user interest from the rating data (Equation 7) and the user social network (Equation 10). A large value of $\alpha$ indicates that we are more inclined to mine the rating data for user interest. A small value of $\alpha$ indicates that we are more inclined to have user interest close to the interest of the user's friends. Figure 9 shows the impacts of $\alpha$ in our method. We observe that $\alpha$ has a significant effect on recommendation results: as $\alpha$ increases, MAE decreases and Recall increases at first, but when $\alpha$ surpasses a certain threshold (0.01), MAE begins to increase and Recall begins to decrease. This phenomenon shows fusing the two aspects generates more accurate user interest than only considering rating data or social information does.

\subsection{Performance Comparison}

In this section, we use different training data $R_{\text {train }}(25 \%, 50 \%$ and $75 \%)$ to evaluate the performance.

Different training data. Table 1 shows comparison results. We observe that in all experiments, our four methods $\left(\mathrm{CSR}_{C F}^{S}, \mathrm{CSR}_{M F}^{S}, \mathrm{CSR}_{M F^{+}}^{-S}\right.$ and $\mathrm{CSR}_{M F^{+}}^{S}$ ) are more accurate than other methods. Traditional memory-based algorithms, i.e., Item-based has the worst performance. PMF performs much better than item-based algorithm, proving the advantage of latent factor model. SocialMF and SoReg have almost the same accuracy, both superior to $P M F$, proving the effectiveness of incorporating social information, but SoReg is a bit lower because it uses better social regularization term. LLR outperforms SoReg, proving that better than social information, category and keyword contribute more to moding user personalized behavior. Note that $\operatorname{CSR}_{M F}^{S}$ performs better than $C S R_{C F}^{S}$, which shows $P M F$ is more efficient than item-based in filling in $S ; C S R_{M F^{+}}^{S}$ performs better than $C S R_{M F}^{S}$, which shows the effectiveness of our neighbor-based approach in improving the sparsity of $S$; $C S R_{M F^{+}}^{S}$ performs better than $C S R_{M F^{+}}^{-S}$, which shows the effectiveness of the user social network in shaping user interest vector. On $25 \% R_{\text {train }}$, our method decreases the prediction error (MAE/Recall) as high as $6.99 \% / 6.04 \%$ in contrast to $L L R$. On $50 \% R_{\text {train }}$, the corresponding improvements are $9.64 \% / 8.98 \%$. On $75 \% R_{\text {train }}$, the corresponding improvements are $11.9 \% / 10.48 \%$.

Different users and items. In order to make a more detailed analysis, we evaluate how our method performs for different users and items. Users are grouped into 5 classes: "1-20", "20-40", "40-80", "80-160" and ">=160", denoting how many ratings a user has in the training data. Items are grouped into 5 classes: "1-20", "20-40", "40-80", "80-160", "160-320" and " $>=320$ ", denoting how many ratings every item has in the training data. From Figure 10 and 11 , we observe that the performance of all the methods is improved gradually with the number of users' and items' observed ratings increasing. We also observe our method outperforms other methods in all the user and item groups, especially when few user ratings or few item ratings are given. Actually, when few ratings are observed, our method can find the accurate tag-keyword relation matrix since tags are selected by multiple users and keywords are shared by multiple items, proving the outstanding ability of our method to handle sparse data.

\footnotetext{
${ }^{3}$ http://math.nist.gov/javanumerics/jama/
} 
Table 1. Performance comparison on Different Training Data

\begin{tabular}{|c|c|c|c|c|c|c|c|c|c|c|}
\hline Training Data & Metrics & Item-based & PMF & SocialMF & SoReg & LLR & $\mathrm{CSR}_{C F}^{S}$ & $\mathrm{CSR}_{M F}^{S}$ & $\mathrm{CSR}_{M F^{+}}^{-S}$ & $\mathrm{CSR}_{M F^{+}}^{S}$ \\
\hline \multirow[b]{2}{*}{$25 \%$} & $\begin{array}{c}\text { MAE } \\
\text { Improve }\end{array}$ & $\begin{array}{l}0.2477 \\
1998 \%\end{array}$ & $\begin{array}{l}0.2269 \\
1265 \%\end{array}$ & $\begin{array}{l}0.2184 \\
924 \%\end{array}$ & $\begin{array}{l}0.2163 \\
8.37 \%\end{array}$ & $\begin{array}{l}0.2131 \\
6.99 \%\end{array}$ & 0.2074 & 0.2031 & 0.2009 & 0.1982 \\
\hline & $\begin{array}{c}\text { Recall@10 } \\
\text { Improve }\end{array}$ & $\begin{array}{l}0.2604 \\
17.93 \%\end{array}$ & $\begin{array}{l}0.2757 \\
11.39 \%\end{array}$ & $\begin{array}{l}0.282 \\
8.9 \%\end{array}$ & $\begin{array}{l}0.2842 \\
8.06 \%\end{array}$ & $\begin{array}{l}0.2896 \\
6.04 \%\end{array}$ & 0.2956 & 0.3003 & 0.3022 & 0.3071 \\
\hline \multirow[b]{2}{*}{$50 \%$} & $\begin{array}{c}\text { MAE } \\
\text { Improve }\end{array}$ & $\begin{array}{c}0.2035 \\
20.29 \%\end{array}$ & $\begin{array}{c}0.1930 \\
15.96 \%\end{array}$ & $\begin{array}{c}0.1914 \\
15.25 \%\end{array}$ & $\begin{array}{l}0.1906 \\
14.9 \%\end{array}$ & $\begin{array}{l}0.1795 \\
9.64 \%\end{array}$ & 0.1708 & 0.1663 & 0.1641 & 0.1622 \\
\hline & $\begin{array}{c}\text { Recall@10 } \\
\text { Improve }\end{array}$ & $\begin{array}{l}0.2867 \\
18.52 \%\end{array}$ & $\begin{array}{l}0.2963 \\
14.68 \%\end{array}$ & $\begin{array}{l}0.2986 \\
13.8 \%\end{array}$ & $\begin{array}{l}0.3035 \\
11.96 \%\end{array}$ & $\begin{array}{l}0.3118 \\
8.98 \%\end{array}$ & 0.3284 & 0.3312 & 0.3347 & 0.3398 \\
\hline \multirow{2}{*}{$75 \%$} & $\begin{array}{c}\text { MAE } \\
\text { Improve }\end{array}$ & $\begin{array}{l}0.1919 \\
26.42 \%\end{array}$ & $\begin{array}{l}0.1891 \\
25.33 \%\end{array}$ & $\begin{array}{c}0.1823 \\
22.55 \%\end{array}$ & $\begin{array}{c}0.1803 \\
21.69 \%\end{array}$ & $\begin{array}{l}0.1580 \\
11.9 \%\end{array}$ & 0.1511 & 0.1475 & 0.1450 & 0.1412 \\
\hline & $\begin{array}{c}\text { Recall@10 } \\
\text { Improve }\end{array}$ & $\begin{array}{c}0.2968 \\
22.13 \%\end{array}$ & $\begin{array}{c}0.2998 \\
20.91 \%\end{array}$ & $\begin{array}{c}0.3063 \\
18.35 \%\end{array}$ & $\begin{array}{c}0.312 \\
16.18 \%\end{array}$ & $\begin{array}{l}0.3281 \\
10.48 \%\end{array}$ & 0.352 & 0.3541 & 0.3689 & 0.3625 \\
\hline
\end{tabular}

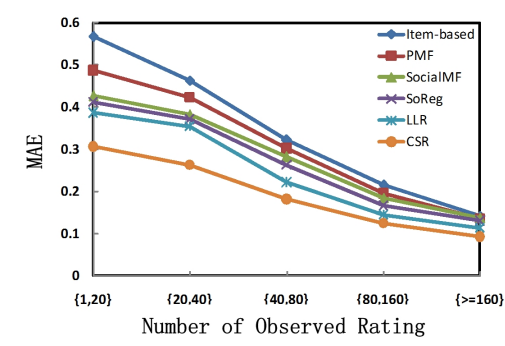

(a) MAE on Different User Rating scales

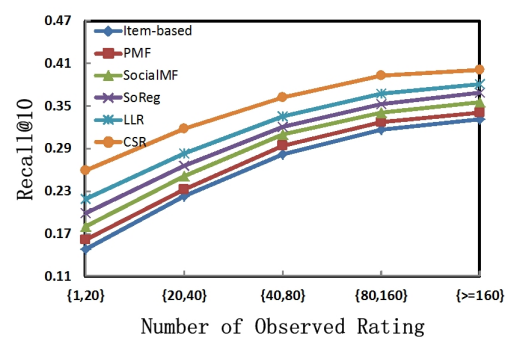

(b) Recall on Different User Rating scales

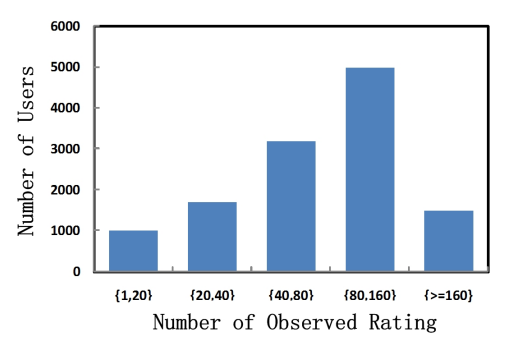

(c) Distribution of Testing Data (User)

Figure 10. Performance Comparison on Different Users (75\% Training Data)

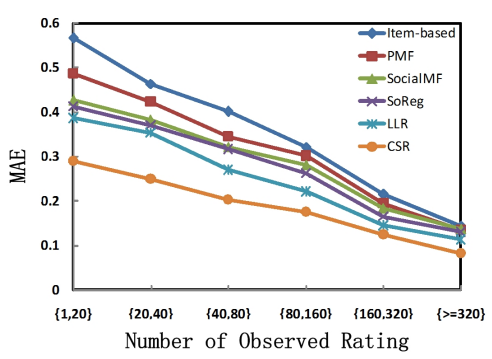

(a) MAE on Different Item Rating scales

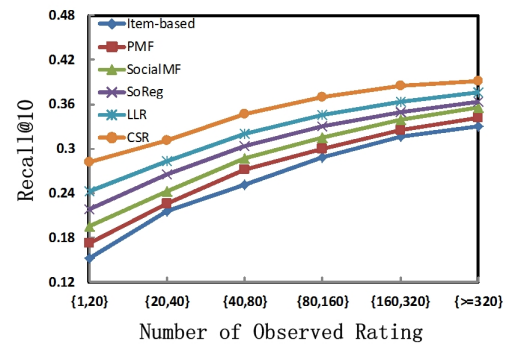

(b) Recall on Different Item Rating scales

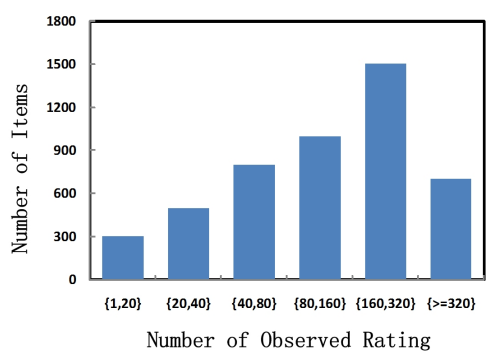

(c) Distribution of Testing Data (Item)

Figure 11. Performance Comparison on Different Items (75\% Training Data) 

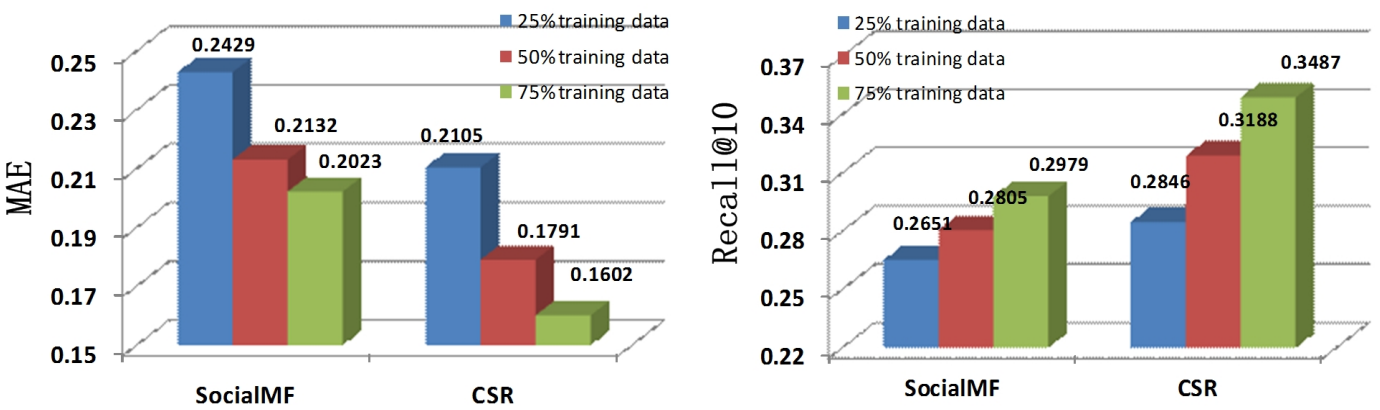

(a) New users
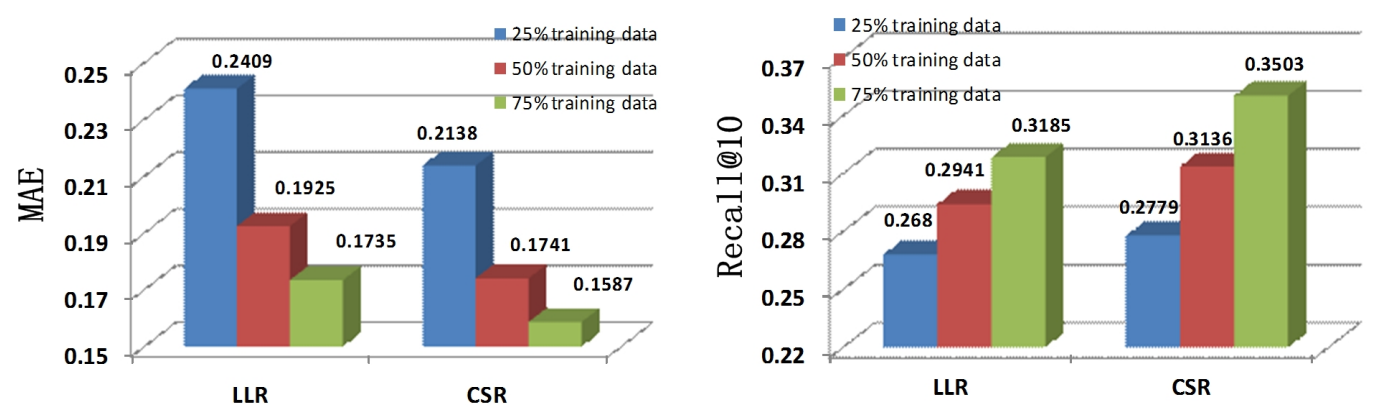

(b) New items

Figure 12. Cold Start Recommendation

\subsection{Cold Start Recommendation}

In this section we analyze the capability of our method on cold start recommendations for new users and new items.

New users. Out of the baselines, SocialMF can deal with new users by incorporating the connections in social network. Figure 12 (a) compare the accuracy of our method and SocialMF on $R_{\text {new }}^{U}$. Results show that our method performs better on both metrics, which shows that our method handles new users better than SocialMF.

New items. Similar to our method, LLR can make recommendation for new items by incorporating item's keywords. Figure 12(b) compare the accuracy of our method and $L L R$ on $R_{\text {new }}^{U}$. We observe that our method outperforms $L L R$ in all the experiments, which shows that our method handles new items better than $L L R$.

New users and new items. One special case is that the user and item both do not exist in the previous rating matrix. All the baselines cannot deal with this situation. But our method can still make recommendations by considering the relations between user's tags and item's keywords. When using $25 \% R_{\text {train }}$, MAE and Recall on $R_{\text {new }}^{U I}$ are 0.2121 and 0.2795 . When using $50 \% R_{\text {train }}$, the corresponding values are 0.1826 and 0.3066 ; when using $75 \% R_{\text {train }}$, the corresponding values are 0.1631 and 0.3408 .

The comparison experiments in this section verify that our method achieves good performance on cold start recommendation for new users and new items.

\section{CONCLUSION}

In this paper, we study the explanation and cold start problems in recommender systems based on collaborative filtering. By incorporating content-based information and social information, we proposed a hybrid method which uses user-item ratings to build content association between users and items based on three factors-user interest in selected tags, tag-keyword relation and item correlation with extracted keywords, and then recommends the items 
with high similarity in content to users. In order to perform the content association, two novel methods (neighborbased approach and 3-factor matrix factorization) and a social regularization are proposed. Analysis shows our method can generate explainable recommendation results with simple descriptions, and experiments demonstrate our method improves recommendation accuracy of state-of-the-art CF models for previous users and items with few ratings, as well as cold start users and items with no rating.

In the future, we may conduct online A/B-tests based experiments to investigate the effect of description information on recommending the items when a user is browsing a website, since our analysis is a simple discussion and our present offline experiments cannot quantify exactly how much effect the explanation has on users' acceptance of the recommended items.

\section{Acknowledgement}

This work is supported by National Science Foundation of China under its General Projects funding \# 61170232 , Research Initiative Grant of Sun Yat-Sen University (Project 985), Australian Research Council (ARC) Discovery Project DP150104871, Ministry of Education Funds for Innovative Groups \#241147529. The corresponding author is Hong Shen.

\section{References}

[1] J. D. M. Rennie, N. Srebro, Fast maximum margin matrix factorization for collaborative prediction, in: Proceedings of the 22nd international conference on Machine learning, ICML '05, ACM, New York, NY, USA, 2005, pp. 713-719. URL: http://doi . acm.org/10.1145/1102351.1102441. doi:10.1145/1102351.1102441.

[2] R. Salakhutdinov, A. Mnih, Probabilistic matrix factorization, in: J. Platt, D. Koller, Y. Singer, S. Roweis (Eds.), Advances in Neural Information Processing Systems 20, MIT Press, Cambridge, MA, 2008, pp. 1257-1264.

[3] T. Hofmann, Latent semantic models for collaborative filtering, ACM Trans. Inf. Syst. 22 (2004) 89-115.

[4] L. Baltrunas, B. Ludwig, F. Ricci, Matrix factorization techniques for context aware recommendation, in: Proceedings of the fifth ACM conference on Recommender systems, RecSys '11, ACM, New York, NY, USA, 2011, pp. 301-304. URL: http://doi.acm.org/10.1145/2043932.2043988. doi:10.1145/2043932.2043988.

[5] S. Rendle, Z. Gantner, C. Freudenthaler, L. Schmidt-Thieme, Fast context-aware recommendations with factorization machines, in: Proceedings of the 34th international ACM SIGIR conference on Research and development in Information Retrieval, SIGIR '11, ACM, New York, NY, USA, 2011, pp. 635-644. URL: http://doi.acm.org/10.1145/2009916.2010002. doi:10.1145/2009916.2010002.

[6] C. Palmisano, A. Tuzhilin, M. Gorgoglione, Using context to improve predictive modeling of customers in personalization applications, Knowledge and Data Engineering, IEEE Transactions on 20 (2008) 1535-1549.

[7] G. Adomavicius, A. Tuzhilin, Context-aware recommender systems, in: Recommender systems handbook, Springer, 2011 , pp. $217-253$.

[8] H. Ma, H. Yang, M. R. Lyu, I. King, Sorec: social recommendation using probabilistic matrix factorization, in: Proceedings of the 17th ACM conference on Information and knowledge management, CIKM '08, ACM, New York, NY, USA, 2008, pp. 931-940. URL: http://doi.acm.org/10.1145/1458082.1458205. doi:10.1145/1458082.1458205.

[9] H. Ma, I. King, M. R. Lyu, Learning to recommend with social trust ensemble, in: Proceedings of the 32nd international ACM SIGIR conference on Research and development in information retrieval, SIGIR '09, ACM, New York, NY, USA, 2009, pp. 203-210. URL: http://doi . acm.org/10.1145/1571941.1571978. doi:10.1145/1571941.1571978.

[10] M. Jamali, M. Ester, A matrix factorization technique with trust propagation for recommendation in social networks, in: Proceedings of the fourth ACM conference on Recommender systems, RecSys '10, ACM, New York, NY, USA, 2010, pp. 135-142. URL: http://doi.acm.org/10.1145/1864708.1864736. doi:10.1145/1864708.1864736.

[11] H. Ma, D. Zhou, C. Liu, M. R. Lyu, I. King, Recommender systems with social regularization, in: Proceedings of the fourth ACM international conference on Web search and data mining, WSDM '11, ACM, New York, NY, USA, 2011, pp. 287-296. URL: http://doi.acm.org/10.1145/1935826.1935877. doi:10.1145/1935826.1935877.

[12] I. King, M. R. Lyu, H. Ma, Introduction to social recommendation, in: Proceedings of the 19th international conference on World wide web, WWW '10, ACM, New York, NY, USA, 2010, pp. 1355-1356. URL: http://doi .acm.org/10.1145/1772690.1772927. doi: $10.1145 / 1772690.1772927$.

[13] J. O'Donovan, B. Smyth, Trust in recommender systems, in: Proceedings of the 10th international conference on Intelligent user interfaces, IUI '05, ACM, New York, NY, USA, 2005, pp. 167-174. URL: http://doi.acm.org/10.1145/1040830.1040870. doi: $10.1145 / 1040830.1040870$.

[14] P. Massa, P. Avesani, Trust-aware recommender systems, in: Proceedings of the 2007 ACM conference on Recommender systems, RecSys '07, ACM, New York, NY, USA, 2007, pp. 17-24. URL: http://doi.acm.org/10.1145/1297231.1297235. doi:10.1145/1297231.1297235.

[15] N. Tintarev, J. Masthoff, A survey of explanations in recommender systems, in: Proceedings of the 2007 IEEE 23rd International Conference on Data Engineering Workshop, ICDEW '07, IEEE Computer Society, Washington, DC, USA, 2007, pp. 801-810. URL: http://dx.doi.org/10.1109/ICDEW.2007.4401070. doi:10.1109/ICDEW.2007.4401070. 
[16] A. I. Schein, A. Popescul, L. H. Ungar, D. M. Pennock, Methods and metrics for cold-start recommendations, in: Proceedings of the 25th Annual International ACM SIGIR Conference on Research and Development in Information Retrieval, SIGIR '02, ACM, New York, NY, USA, 2002, pp. 253-260. URL: http://doi .acm.org/10.1145/564376.564421. doi:10.1145/564376.564421.

[17] K. Ji, H. Shen, H. Tian, Y. Wu, J. Wu, Two-phase layered learning recommendation via category structure, in: Advances in Knowledge Discovery and Data Mining - 18th Pacific-Asia Conference, PAKDD 2014, Tainan, Taiwan, May 13-16, 2014. Proceedings, Part II, 2014, pp. $13-24$.

[18] S.-T. Park, W. Chu, Pairwise preference regression for cold-start recommendation, in: Proceedings of the Third ACM Conference on Recommender Systems, RecSys '09, ACM, New York, NY, USA, 2009, pp. 21-28. URL: http: //doi . acm.org/10.1145/1639714.1639720. doi:10.1145/1639714.1639720.

[19] K. Zhou, S.-H. Yang, H. Zha, Functional matrix factorizations for cold-start recommendation, in: Proceedings of the 34th International ACM SIGIR Conference on Research and Development in Information Retrieval, SIGIR '11, ACM, New York, NY, USA, 2011 , pp. $315-324$. URL: http://doi .acm.org/10.1145/2009916.2009961. doi:10.1145/2009916.2009961.

[20] M. Sun, F. Li, J. Lee, K. Zhou, G. Lebanon, H. Zha, Learning multiple-question decision trees for cold-start recommendation, in: Proceedings of the Sixth ACM International Conference on Web Search and Data Mining, WSDM '13, ACM, New York, NY, USA, 2013 , pp. $445-454$. URL: http://doi .acm.org/10.1145/2433396.2433451. doi:10.1145/2433396.2433451.

[21] K. Stefanidis, E. Pitoura, P. Vassiliadis, Managing contextual preferences, Information Systems 36 (2011) 1158 - 1180.

[22] G. Adomavicius, A. Tuzhilin, Toward the next generation of recommender systems: A survey of the state-of-the-art and possible extensions, Knowledge and Data Engineering, IEEE Transactions on 17 (2005) 734-749.

[23] H. Zhu, E. Chen, K. Yu, H. Cao, H. Xiong, J. Tian, Mining personal context-aware preferences for mobile users, in: Data Mining (ICDM), 2012 IEEE 12th International Conference on, 2012, pp. 1212-1217. doi:10.1109/ICDM.2012.31.

[24] O. Phelan, K. McCarthy, B. Smyth, Using twitter to recommend real-time topical news, in: Proceedings of the Third ACM Conference on Recommender Systems, RecSys '09, ACM, New York, NY, USA, 2009, pp. 385-388. URL: http://doi.acm.org/10.1145/1639714.1639794. doi:10.1145/1639714.1639794.

[25] X. Su, T. M. Khoshgoftaar, A survey of collaborative filtering techniques, Adv. in Artif. Intell. 2009 (2009) 4:2-4:2

[26] R. Jin, J. Y. Chai, L. Si, An automatic weighting scheme for collaborative filtering, in: Proceedings of the 27th annual international ACM SIGIR conference on Research and development in information retrieval, SIGIR '04, ACM, New York, NY, USA, 2004, pp. 337-344. URL: http://doi.acm.org/10.1145/1008992.1009051. doi:10.1145/1008992.1009051.

[27] J. S. Breese, D. Heckerman, C. Kadie, Empirical analysis of predictive algorithms for collaborative filtering, in: Proceedings of the Fourteenth conference on Uncertainty in artificial intelligence, UAI'98, Morgan Kaufmann Publishers Inc., San Francisco, CA, USA, 1998, pp. 43-52. URL: http://dl .acm.org/citation. cfm?id=2074094.2074100.

[28] B. Sarwar, G. Karypis, J. Konstan, J. Riedl, Item-based collaborative filtering recommendation algorithms, in: Proceedings of the 10th International Conference on World Wide Web, WWW '01, ACM, New York, NY, USA, 2001, pp. 285-295. URL: http://doi .acm.org/10.1145/371920.372071. doi:10.1145/371920.372071.

[29] M. Deshpande, G. Karypis, Item-based top-n recommendation algorithms, ACM Trans. Inf. Syst. 22 (2004) $143-177$.

[30] R. Sinha, K. Swearingen, The role of transparency in recommender systems, in: CHI '02 Extended Abstracts on Human Factors in Computing Systems, CHI EA '02, ACM, New York, NY, USA, 2002, pp. 830-831. URL: http://doi.acm.org/10.1145/506443.506619. doi:10.1145/506443.506619.

[31] P. Pu, L. Chen, Trust building with explanation interfaces, in: Proceedings of the 11th International Conference on Intelligent User Interfaces, IUI '06, ACM, New York, NY, USA, 2006, pp. 93-100. URL: http://doi .acm.org/10.1145/1111449.1111475. doi:10.1145/1111449.1111475.

[32] S. Xu, S. Wu, L. Wang, Personalized semantic ranking for collaborative recommendation, in: Proceedings of the 38th International ACM SIGIR Conference on Research and Development in Information Retrieval, SIGIR '15, ACM, New York, NY, USA, 2015 , pp. 971-974. URL: http://doi .acm.org/10.1145/2766462.2767772. doi:10.1145/2766462.2767772.

[33] W. Guo, S. Wu, L. Wang, T. Tan, Social-relational topic model for social networks, in: Proceedings of the 24th ACM International on Conference on Information and Knowledge Management, CIKM '15, ACM, New York, NY, USA, 2015, pp. 1731-1734. URL: http://doi.acm.org/10.1145/2806416.2806611. doi:10.1145/2806416.2806611.

[34] Y. Moshfeghi, B. Piwowarski, J. M. Jose, Handling data sparsity in collaborative filtering using emotion and semantic based features, in: Proceedings of the 34th International ACM SIGIR Conference on Research and Development in Information Retrieval, SIGIR '11, ACM, New York, NY, USA, 2011, pp. 625-634. URL: http: //doi . acm .org/10.1145/2009916.2010001. doi:10.1145/2009916.2010001.

[35] Z. Deng, J. Sang, C. Xu, Personalized video recommendation based on cross-platform user modeling, in: Multimedia and Expo (ICME), 2013 IEEE International Conference on, 2013, pp. 1-6. doi:10.1109/ICME.2013.6607513.

[36] M. Yan, J. Sang, C. Xu, M. Hossain, Youtube video promotion by cross-network association: @ britney to advertise gangnam style, Multimedia, IEEE Transactions on 17 (2015) 1248-1261.

[37] C. Marlow, M. Naaman, D. Boyd, M. Davis, Ht06, tagging paper, taxonomy, flickr, academic article, to read, in: Proceedings of the Seventeenth Conference on Hypertext and Hypermedia, HYPERTEXT '06, ACM, New York, NY, USA, 2006, pp. 31-40. URL: http://doi .acm.org/10.1145/1149941.1149949. doi:10.1145/1149941.1149949.

[38] A. Hulth, Improved automatic keyword extraction given more linguistic knowledge, in: Proceedings of the 2003 Conference on Empirical Methods in Natural Language Processing, EMNLP '03, Association for Computational Linguistics, Stroudsburg, PA, USA, 2003, pp. 216223. URL: http://dx.doi.org/10.3115/1119355.1119383. doi:10.3115/1119355.1119383. 
Ke $\mathrm{Ji}$ is a Ph.D student of School of Computer and Information Technology in Beijing Jiaotong University, Beijing, China. He received the B.Eng. degree from University of Jinan in 2012. His current research interests include recommender system, information retrieval and machine learning. 
Hong Shen is Professor (Chair) of Computer Science with the University of Adelaide, Australia, and currently also a specially appointed professor with Sun Yat-Sen University, China. He received the B.Eng. degree from Beijing University of Science and Technology, the M.Eng. degree from the University of Science and Technology of China, and the Ph.Lic. and Ph.D. degrees from Abo Akademi University, Finland, all in computer science. He was Professor and Chair of the Computer Networks Laboratory in Japan Advanced Institute of Science and Technology (JAIST) during 2001-2006, and Professor (Chair) of Compute Science at Griffith University, Australia, where he taught for 9 years beginning in 1992. With main research interests in parallel and distributed computing, algorithms, data mining, privacy preserving computing, high performance networks, and multimedia systems, he has published more than $\mathbf{3 0 0}$ papers including over 100 papers in international journals such as a variety of IEEE and ACM transactions. Prof. Shen received many honours/awards including China National Endowed Expert of "Thousand Talents", Chinese Academy of Sciences "Hundred Talents", National Education Commission Science and Technology Progress Award, and Chinese Academy of Sciences Natural Sciences Award. He served on the editorial board of numerous journals and chaired several conferences. 


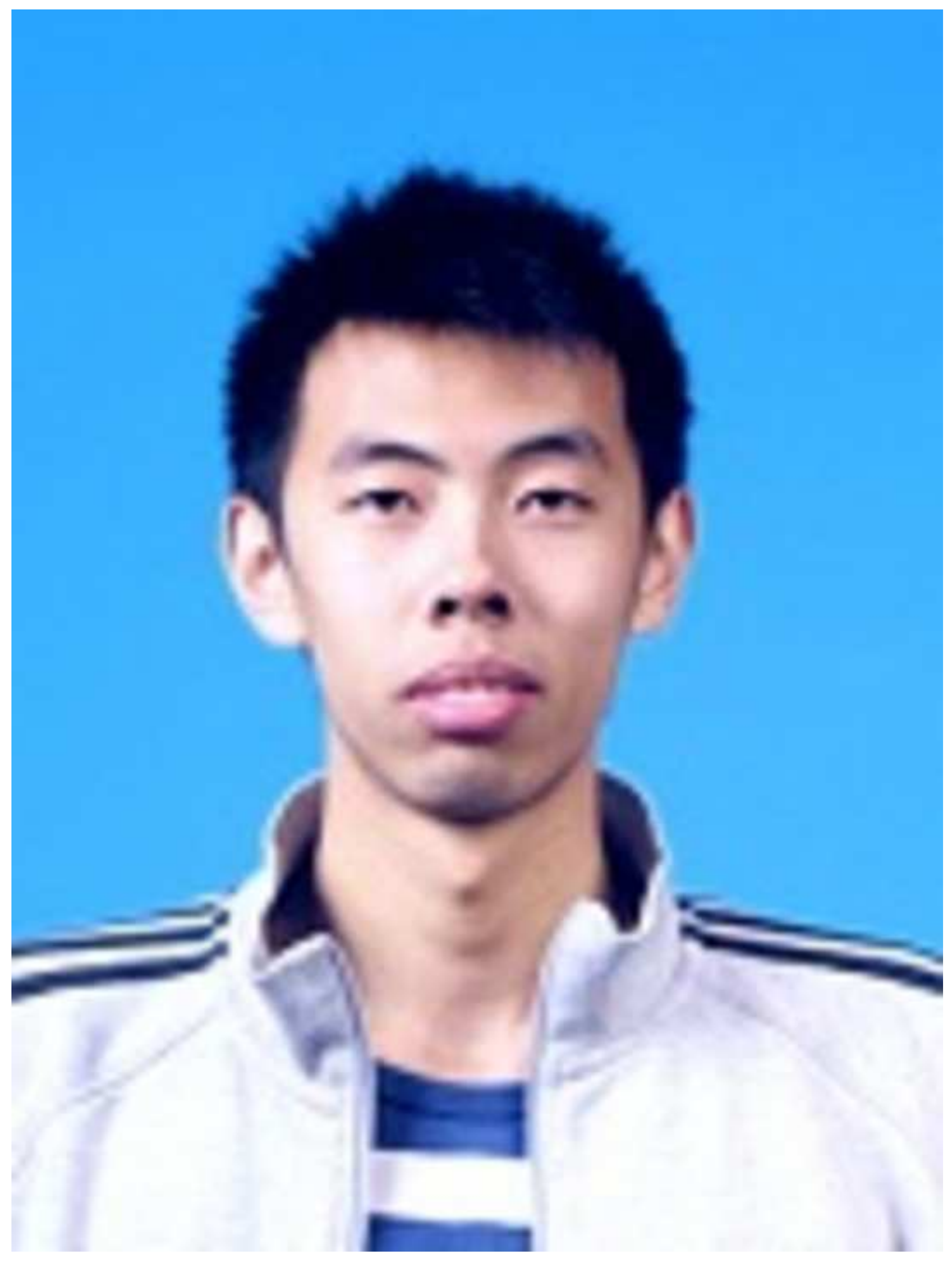

${ }^{*}$ Photo of the author(s

\section{(s)}

\section{Photo of the author(s)}




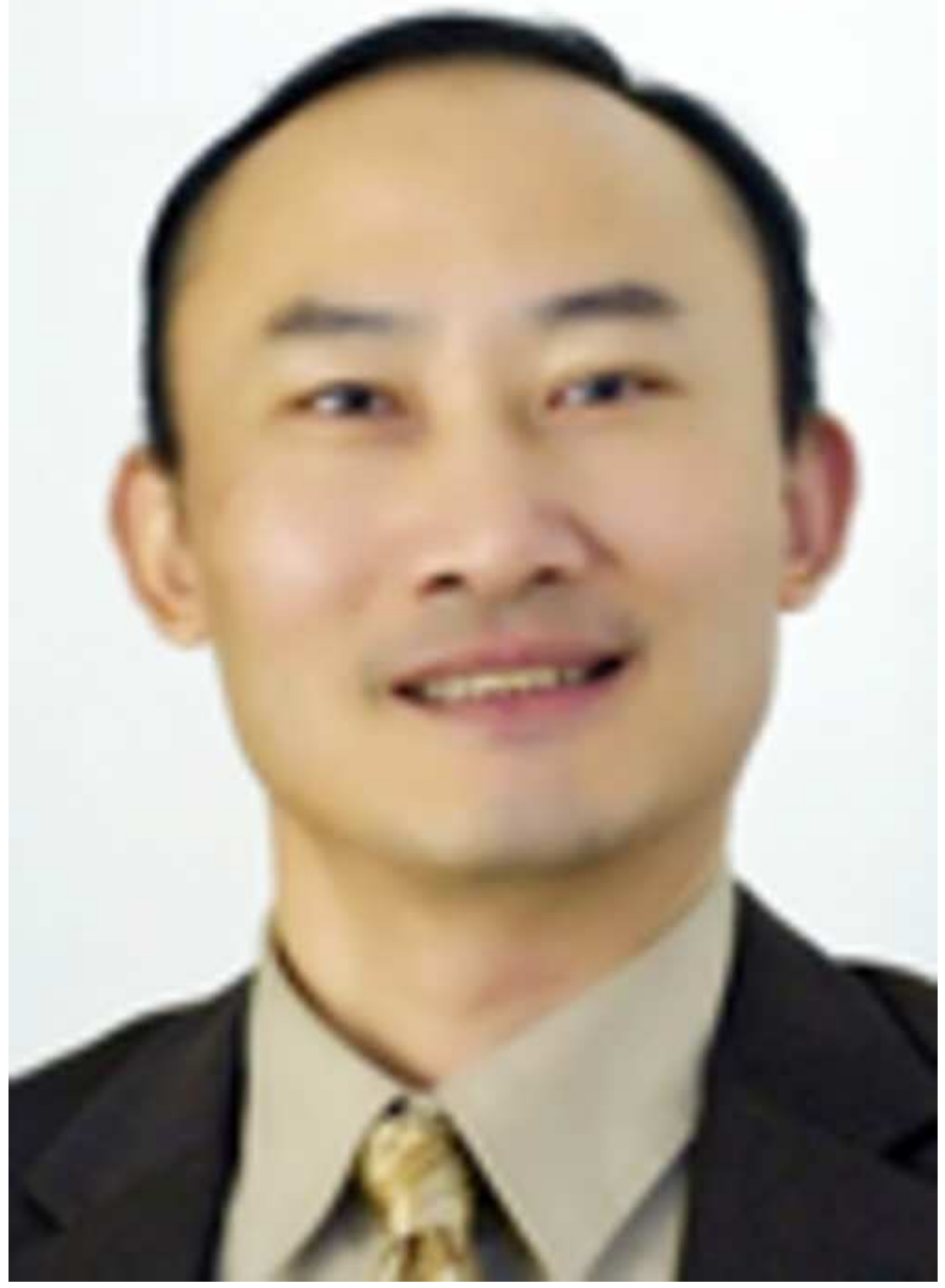

